\title{
Why did Supernova 1054 shine at late times?
}

\author{
J. Sollerman ${ }^{1,2}$, C. Kozma ${ }^{1}$, and P. Lundqvist ${ }^{1}$ \\ 1 Stockholm Observatory, SE-133 36 Saltsjöbaden, Sweden \\ 2 European Southern Observatory, Karl-Schwarzschild-Strasse 2, 85748 Garching bei München, Germany
}

Received 13 July 2000 / Accepted 6 November 2000

\begin{abstract}
The Crab nebula is the remnant of supernova 1054 (SN 1054). The progenitor of this supernova has, based on nucleosynthesis arguments, been modeled as an 8-10 $M_{\odot}$ star. Here we point out that the observations of the late light curve of SN 1054, from the historical records, are not compatible with the standard scenario, in which the late time emission is powered by the radioactive decay of small amounts of ${ }^{56} \mathrm{Ni}$. Based on model calculations we quantify this discrepancy. The rather large mass of ${ }^{56} \mathrm{Ni}$ needed to power the late time emission, $0.06_{-0.03}^{+0.02} M_{\odot}$, seems inconsistent with abundances in the Crab nebula. The late light curve may well have been powered by the pulsar, which would make SN 1054 unique in this respect. Alternatively, the late light curve could have been powered by circumstellar interaction, in accordance with scenarios in which $8-10 M_{\odot}$ stars are progenitors to "dense wind" supernovae.
\end{abstract}

Key words. history and philosophy of astronomy - stars: supernova: general; individual: SN 1054 - ISM: supernova remnants

\section{Introduction}

The Crab nebula is among the best studied objects in the sky. Still, the nature of the progenitor star and many aspects of the explosion remain unclear. The ancient observations of SN 1054, conducted by astronomers in China and Japan, have been analyzed by Clark \& Stephenson (1977). They conclude that SN 1054 was observable in daytime for 23 days and during night for some 650 days past explosion. Great attention has been directed to understand this light curve in terms of supernova theory (Minkowski 1971; Clark \& Stephenson 1977; Chevalier 1977; Pskovskii 1977; Wheeler 1978; Collins et al. 1999, and references therein).

It is now widely accepted that SN 1054 was a corecollapse supernova, primarily due to the presence of the pulsar. That SN 1054 would have been a type Ia supernova (SN Ia), an old suggestion that was recently aired by Collins et al. (1999), is clearly in conflict also with the large amount of mass in the filaments, and in particular with its hydrogen-rich composition. However, the idea that SN 1054 was a normal type II supernova (SN II), as was first suggested by Chevalier (1977), is not unproblematic. The Crab filaments contain only $4.6 \pm 1.8 M_{\odot}$ of material (Fesen et al. 1997) and cruise at merely $\sim 1400 \mathrm{~km} \mathrm{~s}^{-1}$ (Woltjer 1972), which give a kinetic energy an order of magnitude less than the canonical value

Send offprint requests to: J. Sollerman, e-mail: jsollerm@eso.org for core-collapse supernovae, $10^{51}$ ergs. Some of this energy may also originate from the pulsar (Chevalier 1977). As the early observations of SN 1054 indicate a rather luminous explosion, Chevalier (1977) suggested that the missing mass and energy of the Crab resides in a hitherto undetected outer shell. That some material indeed exists outside the visible filaments was recently shown using HST observations (Sollerman et al. 2000).

Nomoto et al. (1982) constructed a detailed model for the progenitor of SN 1054. They argued that the progenitor must have been more massive than $8 M_{\odot}$ in order to leave a neutron star and less massive than $\sim 10 M_{\odot}$ to be consistent with the observed metal abundances. An 8-10 $M_{\odot}$ star would eject very little heavy elements (Nomoto et al. 1982). In particular, it would eject very small amounts of radioactive ${ }^{56} \mathrm{Ni}$, responsible for the late time emission of the supernova (Mayle \& Wilson 1988). In this report we want to draw the attention to the long duration of the light curve of SN 1054, and point out that this cannot be explained within the standard supernova scenario, in which the powering of the emission at these late phases is due to radioactive decay of a very low mass of ${ }^{56} \mathrm{Ni}$.

\section{Discussion}

\subsection{Supernova light curves}

The early light curves of SNe II are powered by the explosion energy slowly diffusing out of the ejecta. The large 
diversity of light curve shapes in this phase (Patat et al. 1994) is largely due to variations in progenitor radius, envelope mass and composition, as well as in the explosion energy itself. At later phases ( $\gtrsim 150$ days), the light curves of SNe II are often powered by the radioactive decay of ${ }^{56} \mathrm{Co} \rightarrow{ }^{56} \mathrm{Fe}$, and then the late evolution is quite uniform (Patat et al. 1994). The ${ }^{56} \mathrm{Co}$ is itself the decay product of ${ }^{56} \mathrm{Ni}$, synthesized in the supernova explosion. The late light curve of the well studied SN 1987A was reproduced by models with $0.07 M_{\odot}$ of ${ }^{56} \mathrm{Ni}$ (Kozma \& Fransson 1998a,b), and most SNe II light curves do follow the decay rate of ${ }^{56} \mathrm{Co}$ (Barbon et al. 1984; Patat et al. 1994). In fact, the luminosity on the light curve tail can be used to determine the mass of ejected ${ }^{56} \mathrm{Ni}$, as has been done for several supernovae (see Sollerman et al. 1998, and references therein).

The radioactive energy from the decay of ${ }^{56} \mathrm{Co}$ is deposited in the supernova ejecta as $\gamma$-rays and positrons. The luminosity on the light curve tail from the $\gamma$-rays is given by $L_{\gamma}=1.2610^{43} F_{\gamma} M_{\mathrm{Ni}} \mathrm{e}^{-t / 111.3} \mathrm{ergs} \mathrm{s}^{-1}$, where $F_{\gamma}$ is the fraction of the $\gamma$-rays trapped in the ejecta, $M_{\mathrm{Ni}}$ is the amount of ${ }^{56} \mathrm{Ni}$ in solar masses, and 111.3 days is the e-folding time for the decay of ${ }^{56} \mathrm{Co}$. The positrons contribute $L_{\mathrm{e}^{+}}=4.4510^{41} M_{\mathrm{Ni}} \mathrm{e}^{-t / 111.3} \mathrm{ergs} \mathrm{s}^{-1}$, assuming they are all deposited locally.

To determine the nickel mass from the observed filter light curves, the $\gamma$-ray trapping and the bolometric correction have to be taken into account. In Fig. 1 we use SN 1987A to illustrate these concepts. There we plot the bolometric light curve of SN 1987A (triangles) from Bouchet \& Danziger (1993). The upper dashed line is given by the expressions above for a nickel mass of $0.07 M_{\odot}$ (Suntzeff \& Bouchet 1990) assuming full trapping of the $\gamma$-rays $\left(F_{\gamma}=1\right)$. This line fits the light curve tail at epochs up to $\sim 300$ days, but later the observed luminosity falls below the luminosity expected for full trapping, due to an increasing leakage of $\gamma$-rays.

The $\gamma$-leakage can be illustrated using a simple model with a central radioactive source, where the deposition $F_{\gamma}=\left(1-\mathrm{e}^{-\tau}\right)$, and the $\gamma$-ray optical depth evolves as $\tau=\left(t / t_{1}\right)^{-2}$ due to the homologous expansion. Here $t_{1}$ is the time when $\tau=1$. For SN 1987A, $t_{1} \sim 610$ days gives a reasonable agreement with the bolometric magnitudes as shown by the dotted curve in Fig. 1. This means that $\sim 60 \%$ of the $\gamma$-rays were trapped at 650 days for SN 1987A.

Also plotted in Fig. 1 is the $V$-band light curve of SN 1987A (square symbols, Suntzeff \& Bouchet 1990). It can be seen that this filter light curve deviates substantially from the bolometric light curve after about 500 days. At these late phases, the ejecta temperature is low and most of the light is instead emitted in the infrared (Kozma \& Fransson 1998a,b).

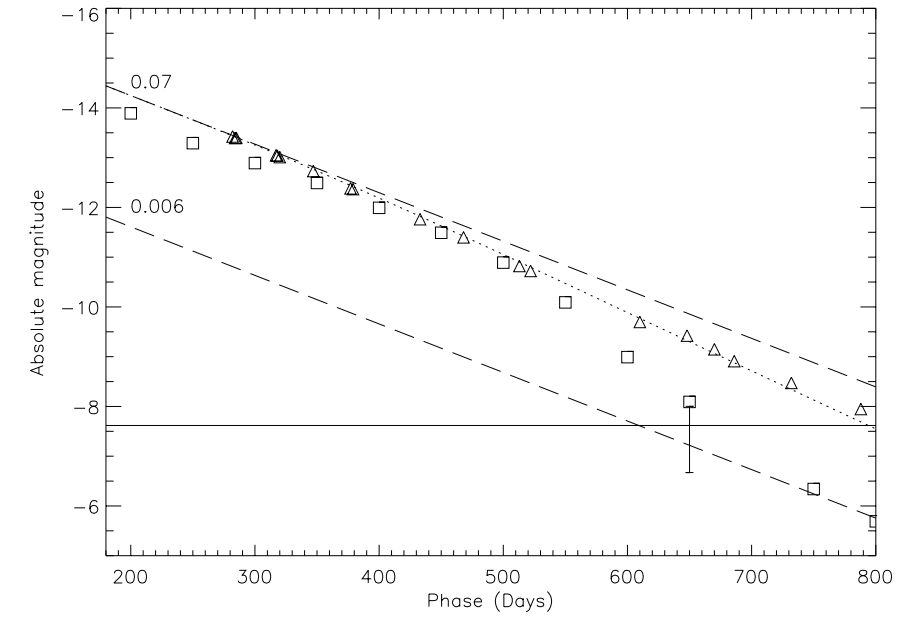

Fig. 1. The upper long dashed line labeled " 0.07 " gives the absolute bolometric magnitude expected from the radioactive decay of $0.07 M_{\odot}$ of ${ }^{56} \mathrm{Ni}$, assuming full trapping. The dotted line allows for $\gamma$-ray leakage with $t_{1}=610$, as explained in the text. This gives a decent fit to the bolometric magnitudes of SN 1987A (50 kpc, $E(B-V)=0.16)$ shown by the triangles (Bouchet \& Danziger 1993). The square symbols show the absolute $V$-band magnitudes for SN 1987A (Suntzeff \& Bouchet 1990), which deviate substantially from the bolometric curve at late phases. The lower dashed line is the absolute bolometric magnitude expected from the radioactive decay of $0.006 M_{\odot}{ }^{56} \mathrm{Ni}$, again assuming full trapping. The horizontal line represents the naked eye detection limit for a source at a distance of $2.0 \mathrm{kpc}$ and with an extinction of $E(B-V)=0.52$, the parameters for SN 1054. The error bar shown at day 650 for this detection limit encapsulates the errors described in the text

\subsection{The late time luminosity of SN 1054}

The Crab is located at a distance of about $2.0 \mathrm{kpc}$ (Trimble 1973) with an extinction of $E(B-V)=0.52$ (Sollerman et al. 2000). From the historical records analyzed by Clark \& Stephenson (1977) we know that SN 1054 faded from visibility some 650 days after discovery. The detection limit for night time observations was estimated to be $5.5 \mathrm{mag}$ by these authors (Clark \& Stephenson 1977). SN 1054 must thus have had an absolute $V$ (actually visual) magnitude of $M_{V}=-7.6$ at this epoch. In Fig. 1 we have indicated this limit together with an error bar that encapsulates distances in the range 1500-2200 pc (Davidson \& Fesen 1985), and an error of \pm 0.04 in $E(B-V)$. As a detection limit of 5.5 magnitudes may be regarded too high, we encapsulate it with conservative limits of $5.5_{-0.3}^{+0.7}$. This is also included in the error bar in Fig. 1.

The Crab progenitor has been modeled as an 8-10 $M_{\odot}$ star (Nomoto et al. 1982). The amount of ${ }^{56} \mathrm{Ni}$ ejected in such an explosion is supposed to be very small. Detailed calculations performed by Mayle \& Wilson (1988) indicate that no more than $0.002 M_{\odot}$ of ${ }^{56} \mathrm{Ni}$ should be ejected from supernovae with progenitors in this mass range. 
The ${ }^{56} \mathrm{Ni}$ eventually decays to ${ }^{56} \mathrm{Fe}$ and thus the current amount of iron probes the mass of ejected nickel. Abundance analyses in the Crab are nontrivial, but indicate a subsolar iron abundance (Davidson 1978, 1979; Henry 1984; Baustista et al. 1996). For an ejecta mass of $4.6 M_{\odot}$, solar abundance corresponds to $0.006 M_{\odot}$ of iron. This is consistent with a low mass of ejected nickel, as suggested by the explosion models of Mayle \& Wilson (1988).

Here we simply want to point out that the naked eye observations of SN 1054 at 650 days seem to be inconsistent with the standard scenario where supernovae from 8-10 $M_{\odot}$ stars are powered at late times by the radioactive decay of very small amounts of ${ }^{56} \mathrm{Ni}$.

Even assuming full trapping and that all of the emitted flux emerges in the visual band, $\left(F_{\gamma}=1, M_{V}=M_{\mathrm{bol}}\right)$, just barely keeps the supernova shining at 650 days past explosion. The lower dashed line labeled "0.006" in Fig. 1 shows a full trapping, bolometric case for this amount of ejected ${ }^{56} \mathrm{Ni}$, and is fairly close to the lower limit of the luminosity needed for naked eye visibility of the supernova at 650 days.

This discrepancy is of course much more pronounced in the more realistic case when the conservative assumptions above are relaxed. For example, assuming that SN 1054 had the same $\gamma$-ray leakage and bolometric correction as $\mathrm{SN} 1987 \mathrm{~A}, \sim 0.05 M_{\odot}$ of ${ }^{56} \mathrm{Ni}$ would be required to reach $M_{V}=-7.6$.

\subsection{Modeling}

Clearly, it is too simplistic to directly compare SN 1054 with SN 1987A. To quantify the discrepancy mentioned above, we have therefore modeled SN 1054 in some detail. Lacking an explosion model, we base our input model on the available observations. We have adopted an ejecta mass of $4.6 M_{\odot}$ (Fesen et al. 1997) and a maximum velocity of $2300 \mathrm{~km} \mathrm{~s}^{-1}$ (Clark et al. 1983; Davidson \& Fesen 1985). We assume that the density and composition are constant throughout the ejecta. We use solar abundances from Cameron (1982) except for helium, for which we use a ratio of the mass fractions of helium and hydrogen, $X(\mathrm{He}) / X(\mathrm{H})=2$ (e.g., Henry 1986).

A central source of radioactive ${ }^{56} \mathrm{Ni}$ is assumed. Calculations have been done for $0.006 M_{\odot}, 0.04 M_{\odot}$ and $0.07 M_{\odot}$ of ${ }^{56} \mathrm{Ni}$. The emission from SN 1054 is modeled in detail from 200 to 800 days. The code is fully described in Kozma \& Fransson (1998a,b). In the decay of ${ }^{56}$ Co, $\gamma$-rays and positrons are emitted, and the thermalization of these is calculated using the Spencer-Fano formalism (Kozma \& Fransson 1992). This time-dependent code successfully reproduces the detailed late time observations of SN 1987A (Kozma 2000).

With this model, we estimate that only $32 \%$ of the $\gamma$-rays were trapped at 650 days for SN 1054. The low trapping is of course due to the low mass of the ejecta, as compared to SN 1987A. In Fig. 2 we plot the light

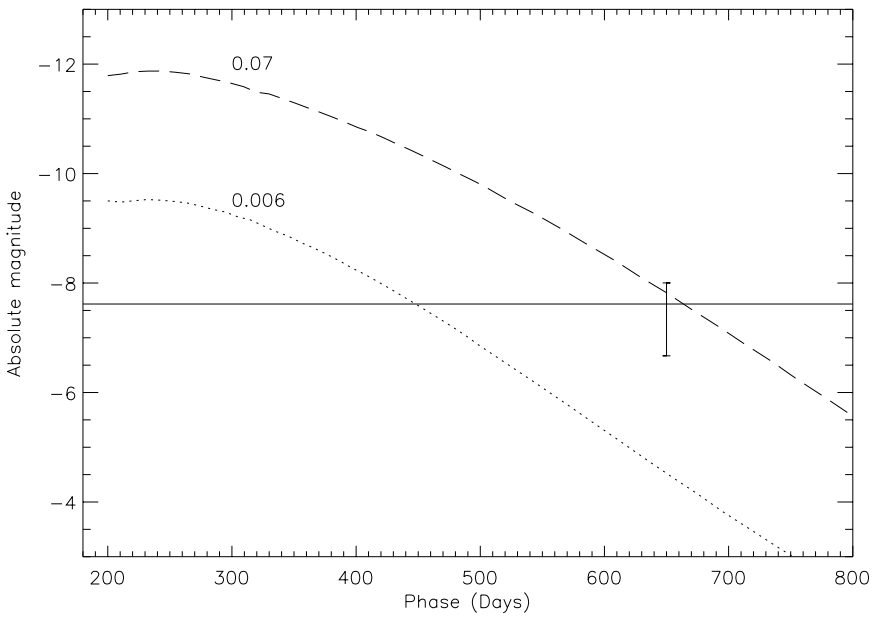

Fig. 2. Absolute $V$-band light curves for the Crab model. The upper curve is for $0.07 M_{\odot}$ of ${ }^{56} \mathrm{Ni}$, while the lower curve is for $0.006 M_{\odot}$ of ${ }^{56} \mathrm{Ni}$. The detection limit is the same as in Fig. 1

curves for the SN 1054 model for two different amounts of ejected ${ }^{56} \mathrm{Ni}$. At 650 days, a smaller amount of the luminosity comes out in the $V$-band for the low nickel-mass model. This is because low amounts of nickel means less heating, and as the temperature of the ejecta decreases the emission is pushed further into the infrared.

From Fig. 2 we directly see that $0.006 M_{\odot}$ of ${ }^{56} \mathrm{Ni}$ could not have provided the observed luminosity at 650 days. Instead, the model with $0.07 M_{\odot}$ of ${ }^{56} \mathrm{Ni}$ (similar to the mass ejected in SN 1987A), comes close to a $V$-magnitude of 5.5 at day 650 . Within the framework of this model, a mass of ${ }^{56} \mathrm{Ni}$ of $0.06_{-0.03}^{+0.02} M_{\odot}$ was required to power the supernova light curve when it faded from night time visibility. This includes the errors estimated above for distance, reddening and naked eye-sensitivity. We have done the same calculations also for a filter similar to the eye sensitivity (Rhodopsin absorption curve, Kitchin 1991), but the deviation from the $V$-filter is small, $0.07 \mathrm{mag}$ for the model with $0.07 M_{\odot}$ of ${ }^{56} \mathrm{Ni}$ at 650 days. Finally, although according to Clark \& Stephenson (1977) there is no reason to question the dates of the Chinese sightings, an error of \pm 20 days in duration would introduce a $30 \%$ error in flux. We note that suggestions for alternative durations, although often based on less direct sources (e.g., Pskovskii 1977; Collins et al. 1999), usually argue for longer visibility of SN 1054, thus enhancing the problem of keeping it shining at late times.

\section{Powering scenarios}

\subsection{Radioactive decay}

If radioactivity alone powered the late time emission, the required mass of ${ }^{56} \mathrm{Ni}$ estimated above, $0.06_{-0.03}^{+0.02} M_{\odot}$, is significantly higher than that obtained in the explosion models by Mayle \& Wilson (1988), $0.002 M_{\odot}$. Although the unknown mechanism of core-collapse explosions makes the existing explosion models rather uncertain, including the exact amount of nickel mass, there are other reasons 
to believe that low mass SNe eject very small amounts of ${ }^{56} \mathrm{Ni}$. Galactic chemical evolution models imply that the amount of iron produced by SNe II is already quite high, and Samland (1998) even suggested that the lower mass limit of stars producing iron should be $11-12 M_{\odot}$, instead of the conventional $10 M_{\odot}$.

Nevertheless, the above arguments cannot conclusively argue that SN 1054 did not eject the $0.06 M_{\odot}$ of ${ }^{56} \mathrm{Ni}$ required to maintain the late time luminosity. We therefore turn the attention to the measured abundances in the Crab nebula.

Actually, only helium is enhanced in this supernova remnant, while the abundances of $\mathrm{C}, \mathrm{N}, \mathrm{O}$ and Fe seem to be (sub)solar (Davidson \& Fesen 1985). In Sect. 2.2 we saw that solar abundance of iron for $4.6 M_{\odot}$ ejecta corresponds to $0.006 M_{\odot}$. The amount of ${ }^{56} \mathrm{Ni}$ required to power the late time light curve, $0.06_{-0.03}^{+0.02} M_{\odot}$, would thus correspond to a present iron abundance which is $9_{-5}^{+4}$ times higher than solar abundance.

If radioactivity powered the supernova, the iron must be locked up in dust. Dust is clearly present in the nebula, and it is nontrivial to determine the dust mass (Sankrit et al. 1998), although estimates seem to indicate that the dust mass is low (Davidson \& Fesen 1985). Note that this scenario would also require large amounts of other metals to be locked up in dust. As the iron-group elements are produced in the very center of the exploding star, we do not expect to find a large amount of iron, while the CNO-abundance has not been enhanced. For example, SN 1987A, which ejected $0.07 M_{\odot}$ of ${ }^{56} \mathrm{Ni}$, also ejected almost 2 solar masses of oxygen. Even the $11 M_{\odot}$ model of Woosley \& Weaver (1995), which ejects $0.07 M_{\odot}$ of ${ }^{56} \mathrm{Ni}$, and only $0.136 M_{\odot}$ of oxygen, provides an oxygen abundance higher than solar. We believe that the scenario in which SN 1054 was powered by $0.06 M_{\odot}$ of ${ }^{56} \mathrm{Ni}$, but where all the nucleosynthesized metals are presently locked up in dust, is not very convincing. We therefore briefly turn our attention to alternative scenarios.

\subsection{The pulsar}

If radioactivity did not power SN 1054 at late times, the obvious candidate is the pulsar that powers the nebula today. Already Chevalier (1977) suggested that the pulsar could contribute to the late time supernova light curve. In fact, unless the bulk of the iron is locked up in dust, and circumstellar interaction was unimportant (see Sect. 3.3), the pulsar would have to make up for all of the late time luminosity, as $0.006 M_{\odot}$ of ${ }^{56} \mathrm{Ni}$ could not have kept the $\mathrm{SN}$ visible for more than $\sim 500$ days (Fig. 2).

There are many ways in which a pulsar can contribute to the optical luminosity of a supernova. Accretion onto the neutron star could be either spherical or in a disk, depending on the angular momentum of the infalling matter. A steady spherical accretion is supposed to reach a maximum Eddington luminosity of $L_{\mathrm{Edd}}=3.510^{38} \mathrm{ergs} \mathrm{s}^{-1}$, and Chevalier (1989) suggested that this scenario might be responsible for the late time luminosity of SN 1054. He worried, however, about the fact that the accretion luminosity would not be able to escape from the vicinity of the neutron star during the first months, as the luminosity is trapped by the inflow (see also Benetti et al. 2000). As shown in Fig. 2, this need not be a problem, as even a low mass of ejected ${ }^{56} \mathrm{Ni}$ is able to keep the supernova shining for some 500 days. If the accretion is mediated via a disk, the luminosity could perhaps be even larger than in the spherical case.

Unknown in these scenarios is the fraction of the luminosity escaping in different bands. In fact, the full Eddington luminosity would have to fall in the visible band to make SN 1054 observable at 650 days (3.5 $10^{38} \mathrm{ergs} \mathrm{s}^{-1}$ corresponds to $\left.M_{\mathrm{bol}}=-7.7\right)$. This appears to be rather unlikely.

Pulsar nebulae in supernovae, with special attention to the Crab, were investigated by Chevalier \& Fransson (1992). Unfortunately, they did not address the question highlighted here, the luminosity of SN 1054 at 650 days. In their scenario, the pulsar powers a bubble that shocks and ionizes the supernova ejecta, and their calculations suggested that $1.5 \%$ of the total pulsar luminosity could be converted to radiation. This would be too low to account for the luminosity of SN 1054 at day 650. However, if the pulsar bubble is a significant source of synchrotron emission (Chevalier 1996), the efficiency might be higher. Another interesting possibility is that the pulsar was born with a very rapid spin period (see Atoyan 1999), and thus a very high initial spin-down luminosity.

In summary, although not investigated in detail, there are several ways in which a pulsar could contribute to the luminosity of supernovae. This has so far never been unambiguously observed. If the pulsar was indeed responsible for the late light curve, this would make SN 1054 a unique case.

\subsection{Circumstellar interaction}

Another mechanism important for supernova light curves is interaction with circumstellar material (CSM). Circumstellar interaction is responsible for the emission at late phases for a number of supernovae, such as SNe 1979C, 1980K, 1988Z, 1993J and 1995N. Such interaction can maintain a SN luminosity of several $\times 10^{38}$ $\operatorname{ergs~s}^{-1}$ for many years (e.g., Chugai et al. 1995). In this respect, we note that the Crab could fit into the scenario of 8-10 $M_{\odot}$ stars being progenitors to the so called "dense wind" supernovae (Chugai 1997), where the supernova ejecta interact with a dense superwind from the progenitor star.

The dense wind must have extended out to $\gtrsim 610^{16} \mathrm{~cm}$ for a maximum ejecta velocity of $v_{\mathrm{ej}} \sim 10^{4} \mathrm{~km} \mathrm{~s}^{-1}$. This means that the wind started $\gtrsim 2\left(v_{\mathrm{ej}} / v_{\mathrm{w}}\right)$ years before the SN breakout. Here $v_{\mathrm{w}}$ is the wind velocity. Comparing with the line fluxes in Chevalier \& Fransson (1994), we find that the late light curve can be explained if $\dot{M} / v_{\text {w }}$ 
was typically in excess of $10^{-5} M_{\odot} \mathrm{yr}^{-1} / 10 \mathrm{~km} \mathrm{~s}^{-1}$. Here $\dot{M}$ is the mass loss rate during the superwind. The swept up wind should in this case now coast freely outside the present nebula. However, no sign of ejecta, or swept up wind, moving at velocities of order $10^{4} \mathrm{~km} \mathrm{~s}^{-1}$ has been identified (e.g., Fesen et al. 1997).

Chugai \& Utrobin (1999) suggested that SN 1054 was similar to the low-energy $\left(\sim 410^{50}\right.$ erg) event SN 1997D (Turatto et al. 1998; Benetti et al. 2000), and that no fast ejecta exist. In this case, circumstellar powering of the late light curve could be more difficult as the luminosity of the circumstellar shock scales as $\propto v_{\mathrm{ej}}^{3}$. Furthermore, in a low-energy explosion, the peak luminosity is more likely to be lower, and Chugai \& Utrobin (1999) therefore suggested that circumstellar interaction was important for SN 1054 also at the early phase. A low-energy explosion model with a circumstellar shell was explored already by Falk \& Arnett (1977), and they found a high efficiency in the conversion of shock energy to visual light during the peak, in accordance with the suggestion of Chugai \& Utrobin (1999). Although there is thus no direct support for circumstellar interaction as the cause for the late emission in the Crab, it is still premature to rule out this possibility.

\section{Summary}

SN 1054, the creation of the Crab nebula and the Crab pulsar, is the typical example of an 8-10 $M_{\odot}$ supernova. Such supernovae are expected to eject only minute amounts of ${ }^{56} \mathrm{Ni}$, and here we demonstrate that SN 1054 could not have been powered by such a small mass of nickel at late times. The required amount of ${ }^{56} \mathrm{Ni}, \sim 0.06 M_{\odot}$, is much larger than suggested by abundance analyses of the Crab, and we therefore discuss alternative solutions. The pulsar may have powered the supernova, which would make SN 1054 unique in this respect. Alternatively, the progenitor of SN 1054 could have had a dense wind and the supernova could then have been powered by circumstellar interaction.

Acknowledgements. We thank the referee for careful reading.

\section{References}

Atoyan, A. M. 1999, A\&A, 346, L49

Barbon, R., Cappellaro, E., \& Turatto, M. 1984, A\&A, 135, 27

Bautista, M. A., Peng, J., \& Pradhan, A. K. 1996, ApJ, 460, 372

Benetti, S., Turatto, M., Balberg, S., et al. 2000, accepted for publication in MNRAS [astro-ph/0010332]

Bouchet, P., \& Danziger, J. I. 1993, A\&A, 273, 451

Cameron, A. G. W. 1982, in Essays in Nuclear Astrophysics, ed. C. A. Barnes, D. D. Clayton, \& D. N. Schramm (Cambridge: Cambridge Univ. Press), 23

Chevalier, R. A. 1977, in Supernovae, ed. D. Schramm

Chevalier, R. A. 1989, ApJ, 346, 847

Chevalier, R. A. 1996, in Supernovae and Supernova remnants, IAU 145, ed. R. McCray, \& Z. Wang

Chevalier, R. A., \& Fransson, C. 1992, ApJ, 395, 540

Chevalier, R. A., \& Fransson, C. 1994, ApJ, 420, 268

Chugai, N. N. 1997, Astron. Rep., 41, 5, 672

Chugai, N. N., Danziger, I. J., \& Della Valle, M. 1995, MNRAS, 276,530

Chugai, N. N., \& Utrobin, V. P. 2000, A\&A, 354, 557

Clark, D. H., \& Stephenson, F. R. 1977, The Historical Supernovae (Pergamon Press)

Clark, D. H., Murdin, P., Wood, R., et al. 1983, MNRAS, 204, 415

Collins, G. W., Claspy, W. P., \& Martin, J. C. 1999, PASP, 111,871

Davidson, K., \& Fesen, R. A. 1985, ARA\&A, 23, 119

Davidson, K. 1978, ApJ, 220, 177

Davidson, K. 1979, ApJ, 228, 179

Falk, S. W., \& Arnett, W. D. 1977, ApJS, 33, 515

Fesen, R. A., Shull, M. J., \& Hurford, A. P. 1997, AJ, 113, 354

Henry, R. B. C. 1984, ApJ, 281, 644

Henry, R. B. C. 1986, PASP, 98, 1044

Kitchin, C. R. 1991, Astrophysical techniques, 2nd ed., 4

Kozma, C. 2000, in Future Directions of Supernova Research: Progenitors to Remnants, ed. S. Cassisi, \& P. Mazzali, in Memorie della Societa Astronomica Italiana, 71, 401 [astro-ph/9903405]

Kozma, C., \& Fransson, C. 1992, ApJ, 390, 602

Kozma, C., \& Fransson, C. 1998, ApJ, 496, 946

Kozma, C., \& Fransson, C. 1998, ApJ, 497, 431

Mayle, R., \& Wilson, J. R. 1988, ApJ, 334, 909

Minkowski, R. 1971, IAU Symp. No. 46, ed. R. D. Davies, \& F. G. Smith (Reidel Dordrecht)

Nomoto, K. Sugimoto, D., Sparks, W. M., et al. 1982, Nature, 299, 803

Patat, F., Barbon, R., Cappellaro, E., \& Turatto, M. 1994, A\&A, 282, 731

Pskovskii, D., Yu 1978, AZh, 55, 737

Samland, M. 1998, ApJ, 496, 155

Sankrit, R., Hester, J. J., Scowen, P. A., et al. 1998, ApJ, 504, 344

Sollerman, J., Cumming, R. J., \& Lundqvist, P. 1998, ApJ, 493, 933

Sollerman, J., Lundqvist, P., Lindler, D., et al. 2000, ApJ, 537, 861

Suntzeff, N. B., Nicholas, B., Phillips, M. M., et al. 1992, ApJ, 384, L33

Suntzeff, N. B., \& Bouchet, P. 1990, AJ, 99, 650

Turatto, M., Mazzali, P. A., Young, T. R., et al. 1998, ApJ, 498, L129

Trimble, V. 1973, PASP, 85, 579

Wheeler, J. C. 1978, ApJ, 225, 212

Woltjer, L. 1972, ARA\&A, 10, 129

Woosley, S. E., \& Weaver, T. A. 1995, ApJS, 101, 181 